\title{
Confusion in interferometer observations
}

\author{
V. S. Artyukh ${ }^{\star}$ \\ Pushchino Radio Astronomy Observatory of the Lebedev Physical Institute of RAS, Pushchino, Moscow region, 142290, Russia \\ and \\ Isaac Newton Institute, Chile, Pushchino Branch, Russia
}

Received 1 April 2003 / Accepted 3 June 2003

\begin{abstract}
The confusion probability distribution in interferometer observations is derived. It is shown that the form of the distribution depends on the correlation between fringe visibilities $V$ and flux densities $S$ of radio sources. Because the $V-S$ correlation depends on the universe model, the evolution of radio source dimensions, and the evolution of the radio luminosity function, the statistical VLBI observations give a new cosmological test to analyse different universe models and evolution models.

An expression for the variance of the confusion distribution is obtained. It is shown that the confusion variance in VLBI observations is much less than that in observations with a single radio telescope.
\end{abstract}

Key words. techniques: interferometric - radio continuum: general

\section{Introduction}

The number of weak radio sources in the reception pattern of an interferometer (or a radio telescope) changes as the interferometer reception pattern is swept across the sky. Continuous recording of the output from the receiving apparatus then produces a trace, which always shows the sum of the effects of the number of radio sources in the reception pattern. This is known as the confusion effect. The measured flux densities of strong sources found on records are affected by the presence of several other, fainter sources in the reception pattern and therefore the confusion causes errors in estimations of the flux densities.

In the meter radio wave range the confusion restricts the sensitivity of observations. For example, the standard deviation of the confusion in observations with the Large Phased Array (LPA) of the Pushchino Radio Astronomy Observatory of the Lebedev Physical Institute is $\approx 1 \mathrm{Jy}$, while the receiver noise level is $\approx 1 \mathrm{mJy}$ (for $T_{S}=300^{\circ}$ at $v=111 \mathrm{MHz}$, $A_{\text {eff }}=3 \times 10^{4} \mathrm{~m}^{2}, \Delta v=2 \mathrm{MHz}, \tau=5^{\mathrm{m}}$ ). The difference is three orders of magnitude, although the LPA is the largest radio telescope in the meter wavelength range $\left(A_{\text {geom }}=7 \times 10^{4} \mathrm{~m}^{2}\right)$. Thus, the confusion restricts the sensitivity of low frequency observations.

At present there are in operation several supergiant radio telescopes such as LOFAR and SKA for low frequency observations. These new generation radio telescopes are intended for observations with very high sensitivity, much higher than we have now. One of the main problems in the creation of these radio telescopes is the suppression of the confusion. For this reason, LOFAR and SKA must be a correlation array.

\footnotetext{
^ e-mail: art@prao.psn.ru
}

A correlation array is a set of interferometers and to estimate the confusion values for observations with the array we must define the theory of confusion in interferometer observations. It is necessary to have a formula to estimate the variance of interferometer response fluctuations due to the confusion.

Investigations of physical conditions in AGNs need low frequency observations of compact radio sources located in the AGNs. It is necessary to create low frequency VLBI systems for these investigations and developing of a theory of the confusion in VLBI observations is currently important.

The confusion effect hinders observations of the individual radio sources but it can give useful information, too. The distribution of signals from a radio telescope gives information about the source counts at weak flux densities when observations of the individual weak source with the radio telescope are not possible due to the confusion (Hewish 1961).

The theory of confusion has been developed by Scheuer (1957). It will be shown that this theory is correct for observations with a single radio telescope. The theory of the confusion in observations of interplanetary scintillating radio sources was developed by Artyukh \& Shishov (1982). In the present paper we consider the confusion effect in interferometer observations.

\section{An interferometer response}

An interferometer response to a completely incoherent radio source looks like (Thompson et al. 1986)

$U(\theta)=\int_{-\infty}^{\infty} I\left(\theta^{\prime}, v_{0}\right) K_{\mathrm{A}}\left(\theta^{\prime}, v_{0}\right) K_{\mathrm{e}}\left(\theta^{\prime}, \Delta v\right) \mathrm{e}^{\mathrm{i} 2 \pi B v_{0}\left(\theta^{\prime}-\theta\right) / c} \mathrm{~d} \theta^{\prime}$ 
Here $I\left(\theta^{\prime}, v_{0}\right)$ is the brightness strip distribution of the radio source at a frequency $v_{0}, K_{\mathrm{A}}\left(\theta, v_{0}\right)$ is the antenna power pattern (normalized to unity at the peak) if the antennas are the same and the product antenna field patterns if the antennas are different, $K_{\mathrm{e}}(\theta, \Delta v)$ is the envelope of the interferometer diagram determined by the band width $\Delta v$ and by the interferometer baseline $B$ (Vitkevich 1952).

Assume that the angular diameter of the radio source is much less than the width of $K_{\mathrm{A}}\left(\theta, v_{0}\right)$ and $K_{\mathrm{e}}(\theta, \Delta v)$; then (1) will be

$$
\begin{aligned}
U(\theta)= & K_{\mathrm{A}}\left(\theta, v_{0}\right) K_{\mathrm{e}}(\theta, \Delta v) \mathrm{e}^{\mathrm{i} 2 \pi B v_{0} \theta / c} \\
& \times \int_{-\infty}^{\infty} I\left(\theta^{\prime}, v_{0}\right) \mathrm{e}^{\mathrm{i} 2 \pi B v_{0} \theta^{\prime} / c} \mathrm{~d} \theta^{\prime}
\end{aligned}
$$

The fringe visibility function is determined as

$V \mathrm{e}^{\mathrm{i} \sigma}=\frac{\int_{-\infty}^{\infty} I(\theta) \mathrm{e}^{\mathrm{i} 2 \pi B v \theta / c} \mathrm{~d} \theta}{\int_{-\infty}^{\infty} I(\theta) \mathrm{d} \theta}$.

Here $V$ and $\sigma$ are the module and phase of the fringe visibility. If the source angular diameter $\Omega_{\mathrm{S}}$ is small (so that $\cos \Omega_{\mathrm{S}} \approx 1$ ) then $\int_{-\infty}^{\infty} I(\theta) \mathrm{d} \theta$ is approximately equal to the flux density $S$ of the radio source. Now (2) may be written as

$U(\theta) \approx S V K_{\mathrm{A}}\left(\theta, v_{0}\right) K_{\mathrm{e}}(\theta, \Delta v) \mathrm{e}^{\mathrm{i}(\sigma+2 \pi \omega \theta)}$

Here $\omega=\frac{B v}{c}$ is the spatial frequency. Strictly speaking the baseline projection $B$ (and hence $V$ and $\sigma$ ) changes with changing $\theta$. In practice $\theta$ changes so little that we can neglect the change in $B$.

As $\theta$ changes in accordance with daily Earth rotation, the function $U(\theta)$ may be presented as a function of time $U(t)$. The interferometer output plots deflection against time; representing $U(t)$ on a complex plane at right angles to the time axis, the record would be a helix of varying radius $S V K_{\mathrm{A}}\left(\theta, v_{0}\right) K_{\mathrm{e}}(\theta, \Delta v)$. The projection of the helix on the real deflection-time plane is the observed record of the interferometer output:

$U(\theta) \approx S V K_{\mathrm{A}}\left(\theta, v_{0}\right) K_{\mathrm{e}}(\theta, \Delta v) \cos (\sigma+2 \pi \omega \theta)$.

Figure 1 shows the response of a phase-switching interferometer to a single radio source. The interferometer reception pattern has the form $K(\theta)=K_{j}(\theta) \cos (2 \pi \omega \theta+\sigma)$, where $K_{j}(\theta)=$ $K_{\mathrm{A}}\left(\theta, v_{0}\right) K_{\mathrm{e}}(\theta, \Delta v)$ (normalized to unity at the peak) is the envelope of the interferometer record and $\cos (2 \pi \omega \theta+\sigma)$ are the interferometer lobes. The amplitude of the response is $V S$. Later on we will write the interferometer response to the radio source in a more compact form:

$U(\theta)=S V K(\theta)$

\section{The probability distribution of interferometer responses to a single radio source}

A radio source with arbitrary flux density $S_{i}$ and with an arbitrary fringe visibility $V_{i}$ located at an arbitrary point in the

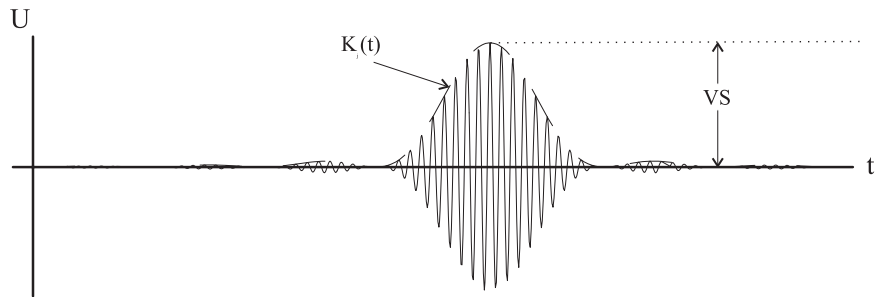

Fig. 1. Phase-switching interferometer response to a single radio source. $K_{j}(t)$ is the envelope of the interferometer record (dashed line).

interferometer reception pattern $K_{i}$ gives the interferometer response $U_{i}$. According to (6) every $U_{i}$ is the product of three random values: $S_{i}, V_{i}$ and $K_{i}$ and therefore $U_{i}$ is a random value too. To obtain the probability distribution of $U_{i}$ we use the standard method of probability theory for coordinate transforms (Mandel \& Wolf 1995). Let introduce two coordinate systems. The first is $K, V, U=S V K$ and the second is $K$, $V, S=U / V K$. After calculating the Jacobian of the transformation from one coordinate system to another we have for a one-dimensional probability distribution

$W(U)=\iint W_{3}\left(K, V, \frac{U}{K V}\right) \frac{\mathrm{d} K}{|K|} \frac{\mathrm{d} V}{|V|}$.

Here $W_{3}\left(K, V, \frac{U}{K V}\right)$ is the three-dimensional probability distribution of the random variables $K_{i}, V_{i}$ and $S_{i}=U_{i} /\left(K_{i} V_{i}\right)$. Because $V$ changes from 0 to 1 , in what follows we omit the module sign. If these variables are statistically independent we get

$W(U)=\iint W_{K}(K) W_{V}(V) W_{S}\left(\frac{U}{K V}\right) \frac{\mathrm{d} V}{V} \frac{\mathrm{d} K}{|K|}$.

The radio source may be in any point of the interferometer reception pattern with the same probability. Taking into consideration the fact that the direct transformation $y=K(\theta)$ is single-valued and the inverse transformation $x=\theta(K)$ is not single-valued we can write

$W_{K}(K) \mathrm{d} K=\int W_{\theta}(\theta) \mathrm{d} \theta=$ const. $\int \mathrm{d} \theta=\frac{\mathrm{d} \Omega}{4 \pi}$,

where $\mathrm{d} \Omega$ is the solid angle inside which $K$ changes from $K$ to $K+\mathrm{d} K$. Substituting (9) in (8), we obtain

$W(U)=\frac{1}{4 \pi} \iint W_{V}(V) W_{S}\left(\frac{U}{K V}\right) \frac{\mathrm{d} V}{V} \frac{\mathrm{d} \Omega}{|K(\Omega)|}$.

The assumption about the statistical independence of the random values streamlines the mathematics but in our case it contradicts the physics. From a physical view the dependence on $V$ and $S$ should be expected (see the Sect. 5). In this case in (10) we must take the two-dimensional probability distribution $W_{2}(V, S)$ instead of the product of the two probability distributions. Then

$W(U)=\frac{1}{4 \pi} \iint W_{2}\left(V, \frac{U}{K V}\right) \frac{\mathrm{d} V}{V} \frac{\mathrm{d} \Omega}{|K(\Omega)|}$.

This is the probability distribution of interferometer responses to a single radio source. 
For comparison, consider the confusion effect in observations with a single radio telescope. Suppose, as before, the radio source is compact: its angular diameter is much less than the width of the antenna reception pattern and $\cos \Omega_{\mathrm{S}} \approx 1$. Then, the radio telescope response is:

$$
\begin{aligned}
U_{\mathrm{A}}(\theta) & =\int I\left(\theta^{\prime}\right) K_{\mathrm{A}}\left(\theta^{\prime}-\theta\right) \mathrm{d} \theta^{\prime} \\
& \approx K_{\mathrm{A}}(\theta) \int I\left(\theta^{\prime}\right) \mathrm{d} \theta^{\prime} \approx S K_{\mathrm{A}}(\theta) .
\end{aligned}
$$

In this formula, $K_{\mathrm{A}}(\theta)$ is the reception pattern of the radio telescope. Using the same standard method of probability theory and taking into account that the random variables $S$ and $K$ are independent, we can write

$$
\begin{aligned}
W_{\mathrm{A}}(U) & =\int W_{2}\left(K_{\mathrm{A}}, \frac{U}{K_{\mathrm{A}}}\right) \frac{\mathrm{d} K_{\mathrm{A}}}{\left|K_{\mathrm{A}}\right|} \\
& =\int W_{K}\left(K_{\mathrm{A}}\right) W_{S}\left(\frac{U}{K_{\mathrm{A}}}\right) \frac{\mathrm{d} K_{\mathrm{A}}}{\left|K_{\mathrm{A}}\right|} .
\end{aligned}
$$

Substituting (9) in (13), we get

$W_{\mathrm{A}}(U)=\frac{1}{4 \pi} \int W_{S}\left(\frac{U}{K_{\mathrm{A}}}\right) \frac{\mathrm{d} \Omega}{\left|K_{\mathrm{A}}(\Omega)\right|}$.

This is the initial formula in Scheuer's theory. It is necessary to emphasize that (14) is correct for observations with a single radio telescope, when a response to a compact source has the form $U_{\mathrm{A}}(\theta)=S K_{\mathrm{A}}(\theta)$. An interferometer response to a source has the form $U(\theta)=\operatorname{VS} K(\theta)$, and the probability distribution of the interferometer response to a source (11) is radically different from (14). The concept of the fringe visibility $V$ is the main concept in interferometry but it is entirely absent in the Scheuer theory. This is why Scheuer's theory is appropriate for the confusion in observations with a single radio telescope, though Scheuer himself thought that he had developed the theory for observations with a interferometer. The obvious exception is the very short-baseline interferometer, when the interferometer response to a radio source is indistinguishable from the single radio telescope response to this source (see Sect. 5).

The probability of meeting a radio source with the flux density from $S$ to $S+\mathrm{d} S$ in an arbitrary direction in the unit solid angle is proportional to the differential source count $n(S)$ :

$W_{S}(S) \mathrm{d} S=\frac{1}{\bar{N}} n(S) \mathrm{d} S$,

here $\bar{N}(S)=\int_{0}^{\infty} n(S) \mathrm{d} S$ is full source number in unit solid angle averaged over all sky.

Suppose $n(S)$ has a power-law form $n(S)=n(1) S^{-\gamma}$ and all variables are statistically independent. This assumption is not correct physically but it simplifies the mathematics. Then from (10) we have

$$
\begin{aligned}
W(U) & =\frac{n(1)}{4 \pi \bar{N}} U^{-\gamma} \int_{0}^{1} V^{\gamma-1} W_{V}(V) \mathrm{d} V \int_{4 \pi} K(\Omega)^{\gamma-1} \mathrm{~d} \Omega \\
& =\frac{n(1)}{\bar{N}}\left\langle V^{\gamma-1}\right\rangle \frac{\Omega_{K}}{4 \pi} U^{-\gamma},
\end{aligned}
$$

where $\Omega_{K}=\int K(\Omega)^{\gamma-1} \mathrm{~d} \Omega$.

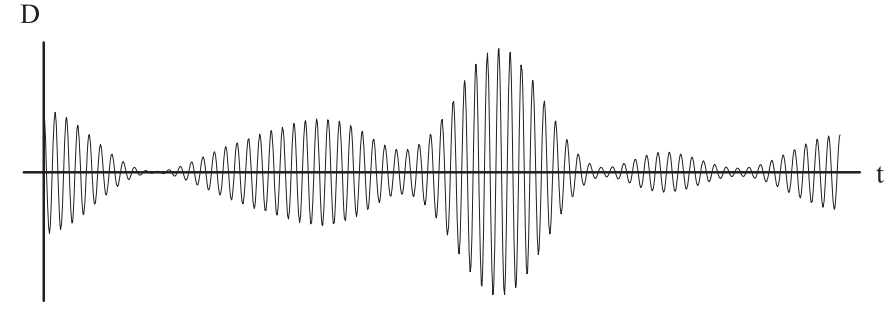

Fig. 2. Schematic drawing of the output from a phase-switching interferometer, showing the passage of several faint radio sources through the interferometer reception pattern.

Thus if $n(S)$ has the power-law form, $W(U)$ has the powerlaw form, too, with the same power index.

Note that numerous source counts, obtained at different frequencies, show that the real number-flux density relations are not power-low functions (Windhorst et al. 1984; Mitchell \& Condon 1985). Accordingly the real $W(U)$ cannot be a powerlow function. This is important because the power-low function has no moments (including variance) since all integrals diverge. For this reason, investigators using the power-low function as the model of $n(S)$ (in spite of the fact that it contradicts observations) are forced to invent artifical methods to remove divergent integrals (see for example Condon 1974).

\section{The probability distribution of interferometer responses to a combined signal from several radio sources}

Figure 2 shows the output from a phase-switching interferometer, where we see the passage of several radio sources through the interferometer reception pattern. At any selected instant of time the interferometer response $D$ is the sum of the responses $U_{i}$ of all the radio sources in the reception pattern:

$D=\sum_{1}^{N} V_{i} S_{i} K\left(\theta-\theta_{i}\right)=\sum_{1}^{N} V_{i} S_{i} K_{i}=\sum_{1}^{N} U_{i}$

where $\theta_{i}$ is the coordinate of the $i$ th radio source. Thus the interferometer response to $N$ radio sources is the sum of $N$ independent random values $U_{i}$ each of which has the same probability distribution $W(U)$. Because of this, we use the infinitely divisible distribution function theory (Lukacs 1970) to obtain the probability distribution of interferometer responses to $N$ radio sources. Let us introduce the characteristic functions

$\tilde{\omega}_{D}(q)=\int_{-\infty}^{\infty} W_{D}(D) \mathrm{e}^{\mathrm{i} q D} \mathrm{~d} D$,

$\tilde{\omega}(q)=\int_{-\infty}^{\infty} W(U) \mathrm{e}^{\mathrm{i} q U} \mathrm{~d} U$,

For the sum of $N$ independent random values we have (Lukacs 1970)

$\tilde{\omega}_{D}(q)=[\tilde{\omega}(q)]^{N}$. 
As the interferometer is scanning the sky, a different number of radio sources fall into the reception pattern at each moment, i.e. $N$ is a random value. But in (20) $N$ cannot be a random value because it is the parameter of the determined function. This is why in (20) we must determine $N$ as the mean value of $N_{i}$ (averaged over all the sky) in the reception pattern: $N=\bar{N} \Omega_{i}=\bar{n}\left(\Omega_{i}\right.$ is the effective beam solid angle of the interferometer reception pattern) and now it is correct to write

$\tilde{\omega}_{D}(q)=[\tilde{\omega}(q)]^{\bar{n}}$.

Suppose $\tilde{\omega}(q)$ can be represented by the expansion in a power series:

$\tilde{\omega}(q)=1+\sum_{1}^{\infty} \frac{\left\langle U^{n}\right\rangle}{n !}(i q)^{n}=1+f(q)$,

here $\left\langle U^{n}\right\rangle=\int_{-\infty}^{\infty} U^{n} W(U) \mathrm{d} U$. Substituting (22) into (21), we get

$\tilde{\omega}_{D}(q)=\mathrm{e}^{\bar{n} \ln [\tilde{\omega}(q)]} \approx \mathrm{e}^{\bar{n} f(q)}=\mathrm{e}^{\bar{n}[\tilde{\omega}(q)-\tilde{\omega}(0)]}$.

Scheuer (1957) has given the original derivation of (23). It is important to note that he did not represent $\tilde{\omega}(q)$ as a power series but the result is the same.

The probability distribution $W_{D}(D)$ can be calculated from $\tilde{\omega}_{D}(q)$ by inverting the Fourier transform (18):

$W_{D}(D)=\int_{-\infty}^{\infty} \tilde{\omega_{D}}(q) \mathrm{e}^{-\mathrm{i} q D} \mathrm{~d} q=\int_{-\infty}^{\infty} \mathrm{e}^{\tilde{n}[\tilde{\omega}(q)-\tilde{\omega}(0)]} \mathrm{e}^{-\mathrm{i} q D} \mathrm{~d} q$.

The expressions (24) and (11) give us the complete solution of the problem.

\section{About the informative capacity of the confusion}

It was noted in the introduction that the confusion could give useful information. As can be seen from (14), $W_{A}(U)$ depends on $W_{S}(S)$, i.e. it depends on the source count $n(S)$. So observations with a single radio telescope give information about the source counts only. It is interesting to note that Hewish (1961) received information about $n(S)$ by analysing interferometer observations (Scott et al. 1961) on the basis of Scheuer's theory. By comparing calculated distributions $W_{D}(D)$ for different models $n(S)$ with the observed $W_{D}(D)$, he chose the suitable $n(S)$. But this result is correct because the observations were made with the short-baseline interferometer $(B=$ $770 \mathrm{~m}, \lambda=1.7 \mathrm{~m}, \Delta v=4 \mathrm{MHz}$ ). The resolution of the interferometer $\approx 7^{\prime}$. Most radio sources have angular diameters $<1^{\prime}$, and therefore they have $V \approx 1$. It is easy to see that the effect of the bandwidth on the fringe envelope of this interferometer is small also. Hewish analysed the envelope of the interferometer output, and in this situation it is possible to write the interferometer response to a compact source in the form $U(\theta) \approx S K_{\mathrm{A}}(\theta)$, where $K_{\mathrm{A}}(\theta)$ is the reception pattern of the greater antenna. Accordingly, for the short-baseline interferometer, when all radio sources are unresolved $(V=1)$, and the interferometer response is similar to the single radio telescope one, Scheuer's theory may be used. Note that in VLBI observations, where most of the radio sources have $V<1$, this theory is not applicable.
What information can we receive from statistical observations with a long-baseline interferometer when radio sources have arbitrary $V$ ? As we see from $(11), W(U)$ depends on the two-dimensional probability distribution $W_{2}(V, S)$, in other words, the $W(U)$ depends on the correlation between fringe visibilities and flux densities of the radio sources. As radio sources are far from the observator, both flux densities of these sources and their angular diameters (and associated $V$ ) must be changed. This implies that the correlation between $V$ and $S$ is to be expected. Different world models give different angular diameter-distance relations, and consequently, different $V-S$ correlations. In addition, the angular diameters of more distant (and hence younger) radio sources must vary due to the evolution of radio source dimensions. Again, flux densities of remote radio sources will be changed owing to the evolution of the radio luminosity function. Hence, calculating the distribution $W_{D}(D)$ we must take into account all these factors. The comparison of the calculated distribution $W_{D}(D)$ with the observed one gives us the possibility to choose suitable evolution models and the world model.

Thus, statistical VLBI observations give us a new cosmological test that can be performed to decide between world models, models of the evolution of radio source dimensions, and models of the radio luminosity functions. It should be noted that it is a purely radio astronomical test and it does not need optical observations, especially very complicated $z$-measuring of weak radio sources.

\section{The variance of interferometer response fluctuations due to the confusion}

As it was noted in the introduction the confusion restricts the sensitivity of low frequency observations. It is desirable to have a simple formula for the estimation of the variance of interferometer response fluctuations due to the confusion. If the second moment of the distribution $W_{D}(D)$ exists, then the confusion variance is

$\Delta D^{2}=\int_{-\infty}^{\infty}(D-\langle D\rangle)^{2} W_{D}(D) \mathrm{d} D$

Unfortunately the expression (25) is very cumbersome to obtain the simple estimation of the confusion variance. This is why we will consider another way to estimate the variance.

Up to here we have considered confusion using the theory of random variables. Now we will analyse the same effect using the theory of stochastic processes. Suppose the output from an interferometer is the stochastic function of time (or coordinates). Let us introduce rectangular coordinates $(x, y)$ on the plane that is a tangent to the interferometer output envelope at the peak. For small angles we can write $\Delta x \approx\left(\alpha-\alpha_{i}\right) \cos \delta_{i}$ and $\Delta y \approx \delta-\delta_{i}$. Here $\alpha_{i}$ and $\delta_{i}$ are the right ascension and the declination of the $i$ th source, $\alpha$ and $\delta$ are the coordinates of the envelope peak. To simplify the problem, we will consider the case when the interferometer reception pattern may be written as $K(x, y)=K_{\alpha}(x) K_{\delta}(y)$. Then the interferometer 
output $D(x, y)$ may be presented as

$$
\begin{aligned}
D(x, y) & =\sum_{1}^{N} V_{i} S_{i} K\left(x-x_{i}, y-y_{i}\right) \\
& =\sum_{1}^{N} A_{i} K_{\alpha}\left(x-x_{i}\right) K_{\delta}\left(y-y_{i}\right) .
\end{aligned}
$$

Here $V_{i}, S_{i}, x_{i}, y_{i}$ are random variables and $K(x, y)$ is the deterministic function. According to Korn \& Korn (1961) the variance of the stochastic function $D(x, y)$ is

$$
\begin{aligned}
\Delta D^{2} & =\bar{N}\left\langle A_{i}^{2}\right\rangle \int_{-\infty}^{\infty} K_{\alpha}^{2}(x) \mathrm{d} x \int_{-\infty}^{\infty} K_{\delta}^{2}(y) \mathrm{d} y \\
& =\bar{N}\left\langle A_{i}^{2}\right\rangle \iint K^{2}(x, y) \mathrm{d} x \mathrm{~d} y,
\end{aligned}
$$

where $\left\langle A_{i}^{2}\right\rangle=\left\langle S_{i}^{2} V_{i}^{2}\right\rangle$.

Suppose there is a correlation between $V_{i}^{2}$ and $S_{i}^{2}$ with the correlation coefficient $R$; then

$$
\left\langle A_{i}^{2}\right\rangle=\left\langle S_{i}^{2}\right\rangle\left\langle V_{i}^{2}\right\rangle+R \sqrt{M_{2}\left(S_{i}^{2}\right) M_{2}\left(V_{i}^{2}\right)} .
$$

Here $\left\langle S^{2}\right\rangle=\frac{1}{N} \int_{0}^{\infty} S^{2} n(S) \mathrm{d} S$ and $\left\langle V^{2}\right\rangle=\int_{0}^{\infty} V^{2} W_{V}(V) \mathrm{d} V$ are the second moments of the probability distributions of random variables $S$ and $V . M_{2}\left(S_{i}^{2}\right)$ and $M_{2}\left(V_{i}^{2}\right)$ are the central second moments of the same distributions.

From physical view points we expect a correlation between $V$ and $S$ but we do not know the character of this correlation. Therefore we cannot use (28) for the estimation of $\Delta D^{2}$. To obtain the approximate estimation of the variance we can neglect the addendum in (28) in comparison with the first term. Really, because $S_{i}^{2}$ and $V_{i}^{2}$ are positive values, the central second moments of distribution of these values are less than the second moments of these distributions and in addition $R \leq 1$. Therefore for approximate estimation of the confusion variance we can use the expression

$\Delta D^{2} \approx \bar{N}\left\langle S^{2}\right\rangle\left\langle V^{2}\right\rangle \iint K^{2}(x, y) \mathrm{d} x \mathrm{~d} y$.

It is obvious that the precise value of $\Delta D^{2}$ differs from (29) by the coefficient that lies between 1 and 2 .

For comparison let us introduce the expression for the confusion variance in observations with a single radio telescope (Artyukh et al. 1973).

$\Delta D^{2}=\bar{N}\left\langle S^{2}\right\rangle \iint K_{\mathrm{A}}^{2}(x, y) \mathrm{d} x \mathrm{~d} y$.

From (29) and (30) one can see that the confusion variance in observations with the interferometer decreased for two reasons: first, the effective interferometer beam width is more narrow than the antenna beam width, and secondly, the coefficient $\left\langle V^{2}\right\rangle$ appears, and it is $<1$. For example, consider the possible interferometer LPA (Russia) - GMRT (India) with the baseline $B \approx 6000 \mathrm{~km}$. The LPA beam width $K_{\mathrm{A}}\left(\theta, v_{0}\right)$ is $48^{\prime} \times 24^{\prime} \mathrm{Secz}$ at $v_{0}=111 \mathrm{MHz}$. If $\Delta v=2 \mathrm{MHz}$ the beam width of $K_{\mathrm{e}}(\theta, \Delta v)$ is $\approx 5^{\prime \prime}$. The baseline of this interferometer is oriented approximately in an east-west direction and consequently instead of the LPA beam width of $48^{\prime}$ we will have the effective interferometer beam width of $5 "$. Therefore the interferometer variance will be nearly three orders less than the confusion variance in observations with LPA. The influence of the second factor will be essential too. The resolution of this interferometer at $111 \mathrm{MHz}$ will be $\sim 0.1^{\prime \prime}$ and the fringe visibility $V$ will be much less than 1 for the vast majority of radio sources. Hence $\left\langle V^{2}\right\rangle$ will be much less than 1 . Thus the confusion in $V L B I$ observations is much weaker than the confusion in observations with a single radio telescope.

\section{The effective beam solid angle of the interferometer reception pattern}

It is necessary to determine the effective beam solid angle $\Omega_{i}$ that we used in the theory. As the Earth is a very good screen of radio radiation, back lobes and far side lobes of the reception pattern of any earth interferometer (or radio telescope) are zero. Furthermore, at very long baselines the interferometer angular field of vision decreases due to the earth's curvature. It follows that in $V L B I$ observations the angular field of vision is small and inside it we see a weak radio source if the source is in the main beam of the interferometer output envelope. This means that the main beam of the interferometer reception pattern must play the main role in the determination of $\Omega_{i}$.

There are different determinations of the effective beam solid angle in the literature. One of them proposed by Condon (1974) is $\Omega_{K}=\int K(x, y)^{\gamma-1} \mathrm{~d} x \mathrm{~d} y$ that appears in (16) as the parameter of $W(U)$ when $n(S)=n(1) S^{-\gamma}$. However, in our opinion this determination is not very good because the real $n(S)$ is not a power-law function. Furthermore, the concept of the angular field of vision must characterize the interferometer (or the radio telescope) itself independent of the investigated objects. But in the current case $\Omega_{K}$ depends on $\gamma$, i.e. on the source counts. Since the real $n(S)$ is a smooth function it is possible to approximate it by several power-law functions (with different $\gamma$ ) at different intervals of flux densities. In this case one telescope will have several angular fields of vision (for all $\gamma$ ) simultaneously. And again, when $\gamma<1, \Omega_{K}$ becomes a divergent integral. Accordingly, this determination is not very good.

In principle, it is possible to take as $\Omega_{i}$ the usual determination of the function dimension via its second moment or simply to take as $\Omega_{i}$ the half power beam width. But in our opinion it will be better to determine $\Omega_{i}$ as

$\Omega_{i}=\iint K^{2}(x, y) \mathrm{d} x \mathrm{~d} y$

because exactly this value is included in expression (29) for the confusion variance in interferometer observations; this $\Omega_{i}$ depends on interferometer parameters only, the integral is not divergent, and with this determination we have the only one angular field of vision for the interferometer.

Acknowledgements. The author wishes to thank V.I. Shishov and N.S. Kardashev for valuable discussion and the language editor for corrections of the paper. This work was supported by the Russian Foundation for Basic Research (project code 01-02-16191a). 


\section{References}

Artyukh, V. S., \& Shishov, V. I. 1982, AZh, 59, 896

Artyukh, V. S., Vitkevitch, V. V., \& Dagkesamanskaya, I. M. 1973, AZh, 50, 924

Condon, J. J. 1974, ApJ, 188, 279

Hewish, A. 1961, MNRAS, 123, 167

Korn, G. A., \& Korn, T. M. 1961, Mathematical Handbook for Scientists and Engineers (New York: Mc Graw-Hill Book Company, Inc.)

Lukacs, E. 1970, Characteristic Functions (London: Griffin)
Mandel, L., \& Wolf, E. 1995, Optical Coherence and Quantum Optics (Cambridge University Press)

Mitchell, K. J., \& Condon, J. J. 1985, AJ, 90, 1957

Scheuer, P. A. G. 1957, Proc. Cambr. Soc., 53, 764

Scott, P. F., Ryle, M., \& Hewish, A. 1961, MNRAS, 122, 95

Thompson, A. R., Moran, J. M., \& Swenson, G. W. 1986, Interferometry and Synthesis in Radio Astronomy (New York: John Wiley \& Sons)

Vitkevitch, V. V. 1952, AZh, 29, 450

Windhorst, R. A., van Heerde, G. M., \& Katgert, P. 1984, A\&AS, 58, 1 